\title{
Depression of Laryngeal Reflexes during Ketamine Anaesthesia
}

\author{
P. A. TAYLOR, R. M. TOWEY
}

British Medical fournal, 1971, 2, 688-689

\section{Summary}

The competence of the laryngeal-closure refiex was assessed in a group of patients having ketamine anaesthesia and found to be depressed. All patients in a control group of conscious volunteers had a competent reflex. The possible dangers of ketamine anaesthesia without endotracheal intubation are discussed.

\section{Introduction}

Ketamine hydrochloride (Ketalar) is a rapid-acting nonbarbiturate general anaesthetic suitable for intravenous or intramuscular use. It is claimed to have unique properties, producing an anaesthetic state characterized by profound analgesia, normal pharyngolaryngeal reflexes, normal skeletal muscle tone, cardiovascular stimulation, and minimal affect on respiration.

The object of this study was to determine whether ketamine hydrochloride caused any depression of pharyngolaryngeal reflexes and thereby assess its safety as a sole agent for anaesthesia in patients with suspected full stomachs-for example, in obstetric and traumatic cases.

\section{Patients and Methods}

Two groups of seven patients were investigated. Group 1 consisted of patients anaesthetized with ketamine. Group 2 comprised fully conscious volunteers acting as controls. Details of the whole procedure were explained to each patient and consent was obtained.

Group 1.-All patients were fit and normotensive undergoing minor peripheral surgery. They were premedicated with scopolamine $0.4 \mathrm{mg}$ intramuscularly one hour before operation and anaesthesia was induced with an initial dose of ketamine $2 \mathrm{mg} / \mathrm{kg}$ body weight. Anaesthesia was maintained by intravenous infusion of ketamine in Hartmann's solution. The infusion was run at a rate sufficient to prevent reflex movement in response to surgical stimulus. With the patient in the supine position $10 \mathrm{ml}$ of Dionosil (a suspension of propyliodone, used in bronchography) was placed on the back of the tongue. This initiated a swallowing reflex in each patient. The patient's chest was then screened with an image intensifier and $x$-ray films were taken at intervals of about one, two, and five minutes.

Group 2.-Each conscious volunteer, also lying supine, had $10 \mathrm{ml}$ of Dionosil placed on the back of the tongue and was asked to swallow. A chest $x$-ray film was then taken. The controls were given $0.4 \mathrm{mg}$ of scopolamine intramuscularly one hour before they swallowed the radio-opaque material.

Department of Anaesthetics, St. Thomas's Hospital, London SE1 P. A. TAYLOR, M.B., F.F.A. R.C.S., Senior Registrar R. M. TOWEY, M.B., CH.B., Registrar

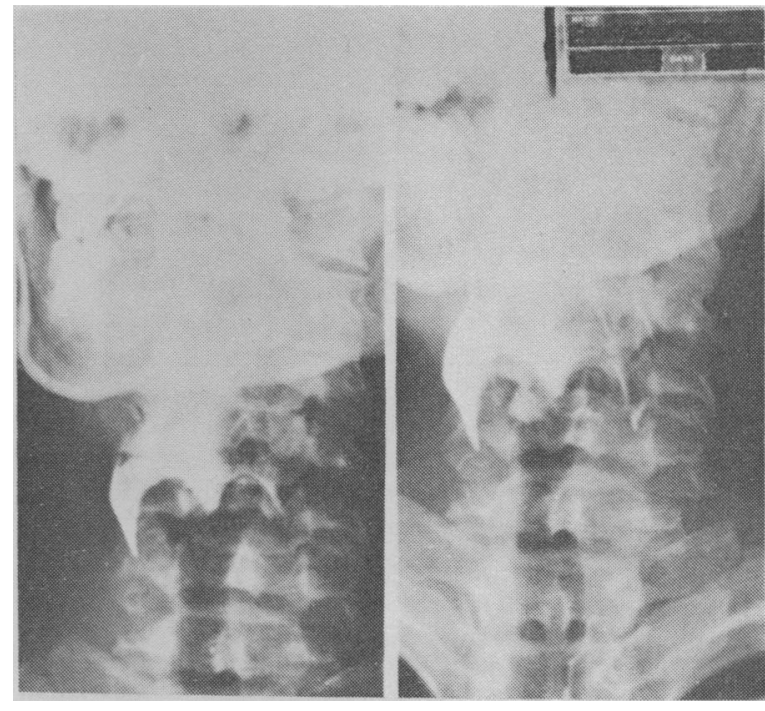

FIG. 1-X-ray film of contrast medium being swallowed by a patient under ketamine anaesthesia. Case from group 1.

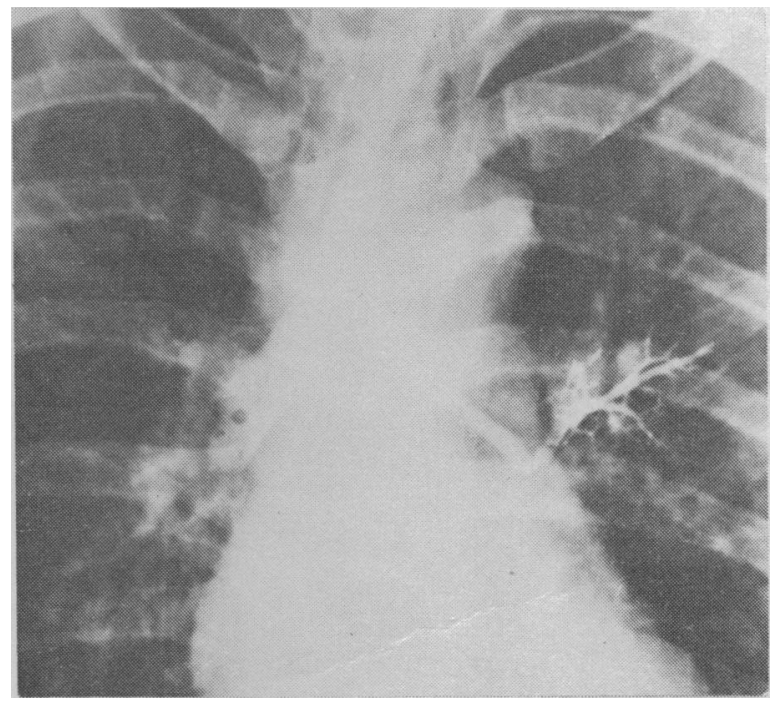

FIG. 2-Later film of patient in Fig. 1. Contrast medium visible in trachea and bronchi.

\section{Results}

Group 1.-All seven patients had clear evidence of opaque material in the lungs on $x$-ray examination. The amount aspirated was sufficient to give good bronchograms in each of these patients (see Figs. 1-3). Aspiration took place into the lungs without any clinical indication of its occurrence. In addition, there was a further patient in whom the study was 


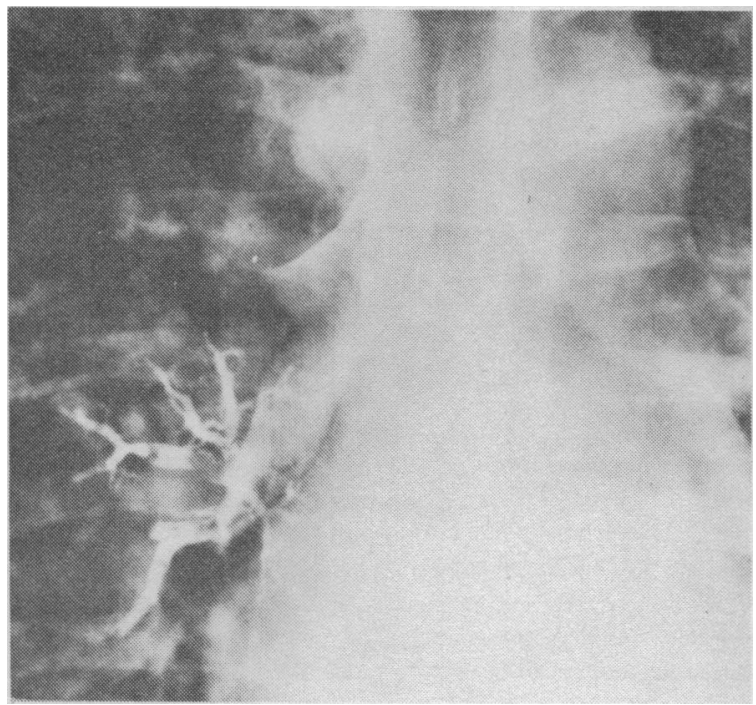

FIG. 3-Typical film showing bronchogram produced by aspiration of contrast medium under ketamine anaesthesia. Case 2 from group 1.

not completed because of the development of partial laryngospasm after only $2 \mathrm{ml}$ of Dionosil.

Group 2.-Each of the seven conscious controls had no $x$-ray evidence of radio-opaque material in the lungs.

\section{Discussion}

None of the conscious patients aspirated contrast medium. This result is supported by the fact that accidential inhalation of radio-opaque dye by patients undergoing oesophageal swallow investigations is rarely reported despite large numbers of such examinations. However, several investigations have shown that the laryngeal-closure reflex is depressed under various forms of conventional anaesthesia (Nunn, 1967; Wise et al., 1969; Healey et al., 1970). In addition, Tomlin et al. (1968) showed that the reflex may not return to normal within two hours of wakening from anaesthesia. This study shows a similar depression during anaesthesia with ketamine.

It would appear, therefore, that ketamine does not differ from conventional anaesthetics in causing depression of pharyngeal and laryngeal reflexes during surgical anaesthesia. The advent of this new type of anaesthetic agent might tempt some clinicians to use ketamine for obstetric anaesthesia on the grounds that its unique properties would make it an ideal agent to use in the presence of a full stomach. These results suggest that it must be accompanied by endotracheal intubation to prevent the risk of silent aspiration.

\section{References}

Healey, T. E. J., Robinson, J. S., and Vickers, M. D. (1970). British Medical fournal, $3,10$.

Nunn, J. F. (1967). Personal communication to Tomlin, et al. (1968).

Tomlin, P. J., Howarth, F. H., and Robinson, J. S. (1968). Lancet, 1, 1402. Wise, C. C., Robinson, J. S., Heath, M. J., and Tomlin, P. J. (1969). British Medical fournal, 2, 540 .

\section{MEDICAL MEMORANDA}

\section{Platelet Response in Fat Embolism and Its Relationship to Petechiae}

\section{E. M. HOARE}

\section{British Medical fournal, 1971, 2, 689-690}

This report describes the case of a patient with the fat embolism syndrome after trauma in whom serial platelet estimations were performed. It is suggested that the petechiae found in the syndrome are more likely to be a sequel to endothelial damage of skin capillaries than to thrombocytopenia, as has been previously suggested (Garner and Peltier, 1967).

\section{Case Report}

A 68-year-old man was admitted to hospital after being knocked down by a car. He was fully conscious and had sustained a simple transverse fracture to his left tibia and fibula and some minor cuts to his head. There was no evidence of brain damage and skull $x$-ray films showed no bony injury. Soon after admission the fracture was manipulated under anaesthesia and an above-knee plaster

Royal Northern Hospital, London N7 6LD

E. M. HOARE, M.B., F.R.C.s., Surgical Registrar. (Present address: New End Hospital, London N.W.3) was applied. He recovered consciousness after the anaesthetic but remained drowsy. Twenty-four hours after the accident he became unconscious and oxygen was administered by mask. A short while later it was noticed that a petechial rash had developed on the upper trunk, being most pronounced over the anterior axillary folds. The arterial $\mathrm{PO}_{2}$ when breathing oxygen by mask at 4 litres

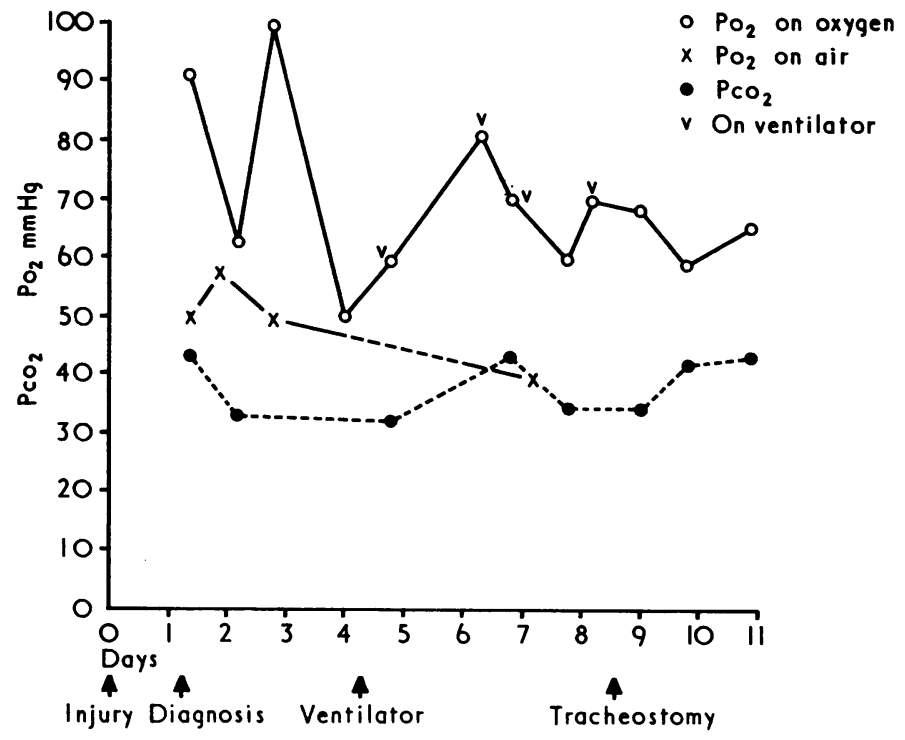

FIG. 1-Arterial $\mathrm{Po}_{2}$ and $\mathrm{PcO}_{2}$. Patient was ventilated from day 4 to day 11 . Blood samples were taken when he had been off the ventilator for at least half an hour, except where indicated. 